\section{TWO NEW AND USEFUL PREPARATIONS FROM CHLOROFORM: CHLORIC ETHER, AND CHLOROFORM JULEP.}

By Thomas Skinner, M.D., Liverpool; Physician to the Liverpool Dispensaries; Fellow of the Obstetrical Society of London; etc.

[Communicated to the Liverpool Medical Institution, April 3rd, 1862.] There are two physical properties possessed by chloroform which, so far as I am aware, are not alluded to in any of our text books on materia medica; namely-1. Its solubility in alcohol, and subsequent miscibility in water; 2. Its miscibility, if not its solubility, in water.

Lately, while engaged experimenting in order to ascer. tain the probable composition of Davenport's chlorodyne, I accidentally discovered the following valuable facts.

1. If chloroform be dissolved in rectified spirit of wine, of specific gravity 838 , at $60^{\circ} \mathrm{Fahr}$. (L. P.), in the proportion of from one to sixteen minims of chloroform in a fluid-ounce, the resulting liquid is entirely and freely miscible with water in all proportions. On adding minim by minim of chloroform to the measure of thirty or thirty-two minims to the ounce of the mixture, the solution ceases to be miscible with water in any proportion; the chloroform spontaneously precipitating in small globules, which ultimately coalesce.

2 . If chloroform be added to distilled or any good drinking water, in the proportion of half a fluid-drachm to a pint (twenty fluid-ounces), and briskly agitated, the resulting liquid is perfectly clear and bright, and no globules of chloroform are precipitated, nor can any be detected with the microscope by a power equal to 250 diameters. On adding more chloroform gradatim, the point of saturation is not arrived at until the proportions are a fluid-drachm to a pint of water. Sixty-four minims to the pint render the mixture quite opalescent, and much of the chloroform is precipitated. At the point of saturation, a drop of any essential oil shaken with the mixture will determine the separation of the chloro. form. If the whole fluid-drachm of chloroform be added at once to a pint of water, and shaken, the result. ing iiquid will not be so clear as when it is added gradually.

Bearing these interesting facts in mind, I resolved to put them to some practical use; and I beg to suggest the propriety of there being two new officinal preparations of chloroform, the names and formula for which shall be as follow :-

Spiritus Formyli Terchloridi (commonly called chloric ether):-Chloroform, $3 \mathrm{v}$; rectified spirit of wine, sp. gr. .838 (L. P.), Oj. Mix. Dose, jss to $3 \mathrm{ij}$.

Mistura Formyli Terchloridi (or chloroform julep):Chloroform, $\overline{5}$ ss; pure water, ()j. Mix thoroughly with brisk agitation, for a minute or two, in a vessel capable of containing double the quantity. Dose, ऊis to $\mathrm{ij}_{\text {. }}$

Either preparation may be prepared from the methylated chloroform; but hitherto I have preferred the unmethylated for administration by the prima via.

A chemical nomenclature has been chosen, in deference to the possible and not improbable fears of the patient.

Remarks. The first preparation requires little to be said about it beyond this: that, although it is already very well known to medical men, and to both wholesale and retail druggists, yet, strange to say, its actual com. position and the method of preparing it have been kept a secret from the profession, and from the members of the drug-trade as a body. I have been informed that Professor Neligan gives a formula for chloric ether, and that there is one wholesale house in London that makes it according to his formula. I can only say that the house alluded to must sell chloroform for chloric ether, as Dr. Neligan's formula is for chloroform, which at one time passed under the cognomen of chloric ether-a less questionable title than that given to the solution of chloroform in spirit of the present day. (See Neligan on Medicines, 2nd ed., 1847, p. 257; and subsequent editions-1851, for instance, p. 301.)

In this town alone there are not two preparations of the so-called chloric ether alike, either in composition or in the invaluable property of being miscible in water. In the April number of the Pharmaceutical Journal, Mr. Norman Tate, of this town, has shown that, out of thirteen specimens of chloric ether obtained by him from various druggists in Liverpool and Birkenhead, there is a difference of $9 \frac{1}{2}$ per cent. between the strongest and the weakest samples. As regards the formula for chloric ether above given, I can aver, from considerable clinical experience, that if it be prepared from bona fide materials, it will equal in medicinal virtue the best chloric ether in the market.

With reference to the other preparation, the chloroform julep, I believe it has hitherto been quite unknown; but the simplicity of its composition, the pleasantness of its taste, the fragrance of its odour, its great and undeniable power as a diffusible stimulant, and its capabilities as a safe and excellent vehicle for adminis. tering other remedies, alkaline, acid, or neutral, bitter, sour, or foetid, need only be alluded to in order to insure it a ready acceptance by the practitioner of medicine. This preparation is reported by some who have tried it to be reckoned a specific for toothache and other painful affections of the mouth and gums. It is simply used as a lotion, wash, or gargle.

The strengths of the foregoing preparations have been made considerably below their respective points of saturation, partly in order to render the dose less irritating to the mouth and throat, and partly to prevent the separation of the chloroform on the addition of an essential oil or such like medicine to a mixture, particularly if prepared from the julep.

In conclusion, whether the above formulæ ever become officinal or not, it is to be hoped that a fixed standard will be given to the spirit of chloroform and chloroform julep in the first or second edition of the coming National Pharmacopæia.

\section{RARE CASES IN MIDWIFERY.}

By Edward Copeman, M.D., Physician to the Norfolk and Norwich Hospital.

[Continued from page 40\%.]

Case xr. Dismemberment. Mrs. W., aged 25, a poor woman residing in Lakenham, supposed to be between seven and ejght months gone in pregnancy, met with some accident on May 28th, 1859, which was soon followed by labour-pains and the escape of liquor amnii. On the following day, however, she was free from pain, and all symptoms of labour passed off. On Monday, the 30th, she again felt pains, but they left her in the evening, and she was able to walk into the town. On Tuesday, she again had slight labour-pains, which left her in the middle of the day, and returned at night; when, finding herself gradually getting worse, she sent for the midwife who had been engaged to attend her. The pains were not very severe, and for some time I understand the midwife was unable to feel the presentation; but at some period of Wednesday morning, June 1st, she dis. covered either a foot or a hand. It proved to be the 489 